\title{
GeO-TOC Project-Reuse of Submarine Cables for Seismic and Geoelectrical Measurements
}

\author{
Junzo Kasahara, ${ }^{*}$ Hiroshi Utada, and Hajimu Kinoshita \\ Earthquake Research Institute, The University of Tokyo, \\ Bunkyo-ku, Tokyo 113, Japan
}

\begin{abstract}
Cooperative efforts by Japanese and U.S. researchers have been initiated to reuse a former submarine telephone cable ("TPC-1," retired in 1991) for undersea scientific measurements. TPC-1 runs from Ninomiya, Japan to Guam, U.S.A., and has a length of ca. $2,700 \mathrm{~km}$ with a strike almost in the $\mathrm{N}$-S direction. A prototype instrument package was successfully installed in northern Japan at depth of $100 \mathrm{~m}$ using a similar coaxial cable. An instrumental package has been built and will be deployed near $30^{\circ} \mathrm{N}$ in 1996 . The instrument has three component accelerometers with 24 bit A/D converters, a hydrophone using analog telemetry, and a quartz thermometer and a quartz pressure sensor with a data length of 16 bits. The data will be transmitted to the Ninomiya station in real-time. Other plans to reuse submarine telephone cables between Naoetsu, Japan and Nakhodka, Russia and between Okinawa, Japan and Guam are being considered.
\end{abstract}

\section{Introduction}

With the recent development of theories and measurement technologies in geophysics, it is known that the structure of the Earth is extremely heterogeneous in terms of seismic velocity, not only with depth, but also horizontally. Seismic wave velocity is the most prominent among the various physical properties used to understand the earth's structure, because it is a function of density, chemical composition, temperature and pressure. Seismic tomographic studies give fine details of the heterogeneity of the Earth by measuring seismic wave velocity (e.g., Dziewonski, 1984). However, most seismic data used to obtain these images were collected on land. Because there are so few submarine seismic stations only a few of which are operated in real-time, the resolution of the earth's structure is still very low for oceanic areas. The low density of submarine observatories is common not only for seismic stations, but also for other geophysical measurements, such as geoelectro-magnetic field measurements, tide gauge, and crustal deformation measurements.

Among the technologies that can be used to install observatories in oceanic areas, the mooring system and the free-fall and pop-up system are the most widely used. For various reasons, e.g., the large volume of data and the desire to detect earthquake

Received April 30, 1992; Accepted May 17, 1995

* To whom correspondence should be addressed. 
precursors, real-time seismic data collection is frequently desirable. For real-time data telemetry, satellite-link buoy systems and the submarine cable systems are the technologies commonly used. However, it is difficult to retrieve data with a high sampling-rate using a satellite-link on the ocean surface, especially in rough seas. In addition, data telemetry sent from the ocean bottom to a surface buoy at a high data transmission rate causes other difficulties. In contrast to a satellite-link buoy system, a submarine cable system might be the most stable tool among the various real-time and high sampling-rate data retrieval systems in the ocean, although the construction cost is high. To overcome this high cost, an alternate method, which reuses abandoned submarine telephone cables for the geophysical measurement, has been proposed by the present authors (Kasahara, 1990) and U.S. scientists (IRIS, 1992).

Due to advances of optical fiber technology, submarine telephone cables using old coaxial technology are being replaced by new ones. There are many coaxial submarine telephone cables world-wide (U.S. Department of Commerce, 1991). Most of these cables will be replaced by optical fibers soon. Constructed in 1964, TPC-1 (Trans Pacific Cable-1) was the first U.S.A.-Japan submarine telephone cable between NinomiyaGuam-Hawaii. This cable system was replaced by TPC-3, which uses optical fibers, in 1991. The Ninomiya-Guam section of TPC-1, which runs nearly in the N-S direction, was donated by cable companies to the scientific community: the University of Tokyo, Japan and IRIS (Incorporated Research Institutions for Seismology), U.S.A., for scientific use. This portion of the cable runs along the Izu-Bonin-Mariana islands where seismicity is high because of the subduction of the Pacific Plate beneath the Philippine Sea plate. This submarine cable will be modified and seismic instruments will be installed on it to give a real-time seismic monitoring system (Fig. 1). In a future plan, other

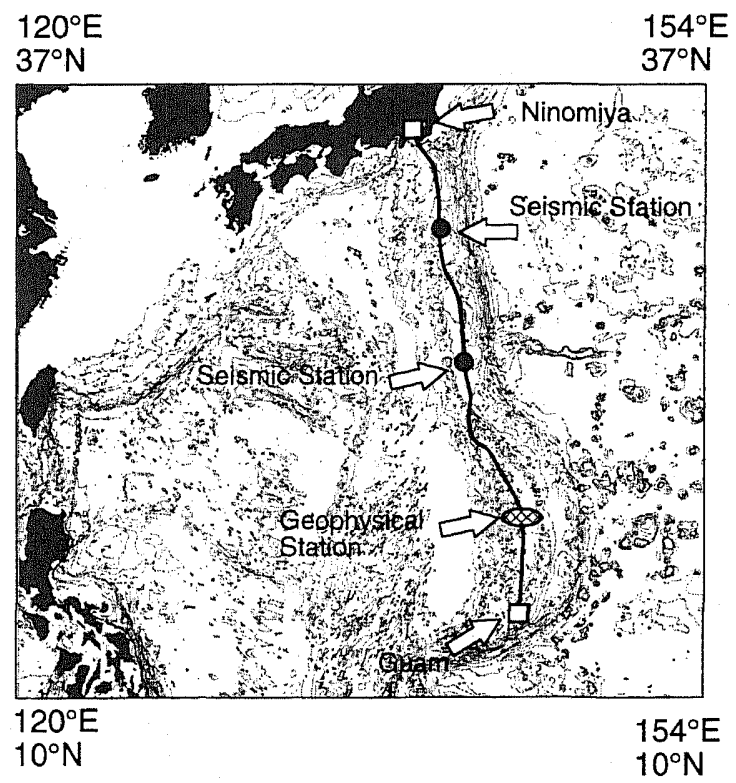

Fig. 1. Location of proposed instruments along "GeO-TOC." 
geophysical sensors will also be installed in the bottom package. The cable will hereafter be called GeO-TOC (Geophysical and Oceanographical Trans Ocean Cable), the name of the Japanese scientific project for reuse of TPC-1.

\section{Reuse of Retired Telephone Cables for Scientific Measurements}

\subsection{Scientific goals}

Plate tectonics explains the major features of seismicity at trenches and surrounding areas. New oceanic plate material is created by oceanic spreading and old oceanic lithosphere subducts at oceanic trenches. The Izu-Bonin-Mariana region is a good place to investigate the plate subduction mechanism in detail. Here the Pacific Plate subducts beneath the Philippine Sea Plate. The dip angle of the subduction is rather gentle in the Izu region and steep in the Bonin-Mariana region (e.g., Kasahara and Tanaka, 1984).

The GeO-TOC cable runs along the fore-arc basin of the Izu-Bonin archipelago and enters the Mariana trough. In contrast to plate subduction, backarc rifting (spreading) seems to be generating a new oceanic plate material. The Mariana trough is thought to be one of the most active sites of backarc spreading. The bathymetry of the Mariana trough at $18^{\circ} \mathrm{N}$ has a topographic resemblance to the mid-Atlantic ridge (Kasahara et al., 1993). Along the axis of the trough, hydrothermal vents were found (Craig et al., 1987), suggesting the existence of ongoing oceanic rifting. The maximum temperature at one of the hydrothermal vents is more than $285^{\circ} \mathrm{C}$. At this point, the GeO-TOC cable runs $80 \mathrm{~km}$ west of the thermal vents.

Among the candidates for geophysical stations along the GeO-TOC cable are an instrument in the Izu region to study the subduction mechanism using seismic data and one in the Mariana trough region to study the nature of hydrothermal activity and its relation to the subduction process.

By continuously monitoring the voltage change in the cable between Guam and Ninomiya, the induced electrical field caused by the magnetic field variation can help to constrain the electrical conductivity distribution in the earth by separating the effects of the solid earth's electrical conductivity from the electrical field generated by ocean currents. In addition to the above measurements, other measurements such as bottom water temperature and pressure, and bottom current will reveal the dynamics of deep-sea currents.

\subsection{General description of "GeO-TOC"}

To reuse an abandoned submarine telephone cable for scientific measurements, it is necessary to examine various factors, such as 1) the life of cable, 2) the upper limit of usable voltage, 3) techniques for instrument deployment, 4) size of instruments, 5) installation sites, and 6) power and data transmission characteristics.

The total length of the GeO-TOC cable is a $2,697 \mathrm{~km}$. The coaxial cable, called $\mathrm{SD}$, has a $2.54 \mathrm{~cm}$ (one inch) diameter for the unarmored segment on the deep sea floor. The TPC-1 cable was previously used for 138 telephone channels, each with a $3 \mathrm{kHz}$ bandwidth, in both directions between Ninomiya and Guam. The $1.052 \mathrm{MHz}$ total bandwidth was divided into the upper band $(660-1,052 \mathrm{kHz})$ to Guam and the lower band $(108-504 \mathrm{kHz})$ to Ninomiya. The cable has 74 repeaters, in which dual vacuum

Vol. 43, No. 5, 1995 
tube amplifiers are used and seven equalizers for every $37.08 \mathrm{~km}$ (20 nautical miles) and every $370.8 \mathrm{~km}$ (200 nautical miles), respectively. The power feed equipment in Guam supplies $4,261 \mathrm{~V} \mathrm{DC}$ with a $370 \mathrm{~mA}$ constant current to the cable. Three thousand, two hundred and fifty-five $\mathrm{V}$ is the voltage drop caused by the repeaters and equalizers and the rest of the voltage is the drop due to cable resistance. The Ninomiya end is grounded to the sea. The maximum voltage allowance of the cable system is $6,000 \mathrm{~V}$, the considering possibilities of an anomalous voltage change during magnetic storms. If a $4 \mathrm{kHz}$ bandwidth is used instead of $3 \mathrm{kHz}$ for each channel, the total number of usable channels will be decrease. By using a $4 \mathrm{kHz}$ bandwidth, however, the cost of receiving equipment will be reduced and $4.8 \mathrm{kbps}$ data can be transmitted through the cable. When a higher data-rate is required, such as acoustic sound from a hydrophone, it is easy to expand the bandwidth from $4 \mathrm{kHz}$ to, say, $64 \mathrm{kHz}$.

Because of the design policy at the time for constructing TPC-1, the life of vacuum tubes was designed to be more than 25 years (AT \& T, personal communication), and the usable life of the coaxial cable seems to be even longer, assuming there is no human or natural damage. There is a trade-off between the usable life and construction cost of the system. If scientists want to install instruments in the mid-ocean at a lower cost, it is better to reuse a submarine cable than lay a new one, but if an extremely large amount of data and a life longer than 25 years are needed, it is necessary to use an optical fiber cable.

\subsection{Reuse of the cable}

The frequency of seismic signals is typically lower than $30 \mathrm{~Hz}$. The dynamic range required for a seismic study needs more than $120 \mathrm{~dB}$. For this reason, it is necessary to use digital data transmission through this cable. When 24 bit A/D conversion with a sampling rate of $100 \mathrm{~Hz}$ is used for seismic signals, the amount of data from one seismometer is $2,400 \mathrm{bps}$. Including some redundancy, $4,800 \mathrm{bps}$ for one seismometer is sufficient, even if the S/N quality of the cable is not so good. This can be obtained with state-of-the-art technology. If the power dissipation from one instrument is similar to that for a repeater, it can be obtained by increasing the supply voltage by several tens of volts.

The first step to obtain undersea measurements using a former submarine telephone cable is to make a prototype instrument and deploy it along a short test cable. The second step is to build the actual instrument to be used for the GeO-TOC cable. The major geophysical quantities to be monitored at the ocean bottom are: 1) seismic waves, 2) the electromagnetic field, 3) undersea-acoustic signals, 4) water temperature, 5) tsunamis, 6) deep-sea ocean currents, and 7) water pressure. Of these, a prototype instrument was designed to measure items 1) to 5), the actual instrument that will be installed in-situ can measure 1) and 3), as well as observe items 4) and 7). These items were selected mainly due to the limits of available budgets and the space for the pressure case. Item 6) was not tested under the current study. The most important among the above items is seismic observation, because earthquake prediction is one of the top priorities in Japanese national research and this needs real-time telemetry. 


\subsection{Prototype instrument}

A prototype instrument was built and has been tested in shallow water, approximately $100 \mathrm{~m}$ depth, off Miyagi Enoshima island, off the northern coast of Japan, in 1993. The total length of the coaxial cable is $3 \mathrm{~km}$. The data collected in the bottom package are transmitted to a PC on the island at $19.2 \mathrm{kbps}$ and control signals can be sent from the island to the data-acquisition unit.

The prototype instrument has several different kinds of sensors: seismometers, magnetometers, a tsunami meter, thermometers inside and outside the package and low-resolution inclinometers. Two different kinds of 3-component seismometer are used: $2 \mathrm{~Hz}$ velocity seismometers (L-22, Mark Product) and force-balance accelerometers (JA-5V-III, JAE). Although the particle velocity of the ground can be obtained by integrating the acceleration records, a velocity seismometer is one of the most reliable seismic sensors for normal seismic measurements. Because a 20-bit CMOS A/D converter was difficult to use at that time, a bipolar 16 bit A/D converter was used. To obtain 20-bit resolution, 16-bit A/D conversion is done twice. First, one 16-bit digital datum is obtained with a low amplification, and then the corresponding analog voltage to this 16-bit datum is subtracted from the original input. The rest of analog voltage amplified 16 times is then converted into digital form by the same 16-bit A/D converter. Using these two data sets, one 20 -bit datum is obtained. However, it was found that the effective data-length is only 18 bits due to the inaccuracy of the $\mathrm{D} / \mathrm{A}$ converter for the subtraction process. A $100 \mathrm{~Hz}$ sampling rate is used for the seismometers. The $14.4 \mathrm{kbps}$ data ( $=24$ bits $\times 6$ channels) from the seismometers are sent to the first 16-bit CPU.

The 3-component fluxgate magnetometers and a proton magnetometer are used for geomagnetic measurements. The proton magnetometer gives a higher accuracy for the magnetic total force and fluxgate magnetometers give vertical and two horizontal magnetic components. The 2-component inclinometers are used to find the inclination angles of the fluxgate magnetometers. A differential hydrophone is a sensor for measuring tsunamis. A quartz thermometer for measuring water temperature gives an accuracy of $0.001^{\circ} \mathrm{C}$. The data rate in the second package is slower than the seismic package. All data are formatted by the second controller with a $19.2 \mathrm{kbps}$ data-rate and then are sent to the PC on the island. The PC stores the data on a DAT (Digital Audio Tape) with double buffers. The $50 \mathrm{~V} \mathrm{DC}$ power is supplied to the bottom package through the cable. The prototype instruments worked successfully with the exception of power supply complexity among several measurements.

\subsection{Design of actual instrument}

The actual instrument (Figs. 2 and 3), which will be deployed at Izu at $30^{\circ} \mathrm{N}$ (see Izu in Fig. 1) along the GeO-TOC cable, has been built. This instrument uses two kinds of acoustic sensors. To observe seismic signals, a 3-component accelerometer is used. The minimum noise level of this accelerometer at periods shorter than $20 \mathrm{~s}$ was found to be about $0.1 \mathrm{mgal}$ (Katao et al., 1990). Considering the results from the prototype instrument, we decided to use three 24-bit A/D converters for this instrument rather than two 16-bit A/D converters. Because seismic signals from microearthquakes contain $20-30 \mathrm{~Hz}$ frequency components, it is necessary to use a sampling rate as fast as $100 \mathrm{~Hz}$. The dynamic range for seismic observations should be greater than $120 \mathrm{~dB}$. Considering

Vol. 43, No. 5, 1995 


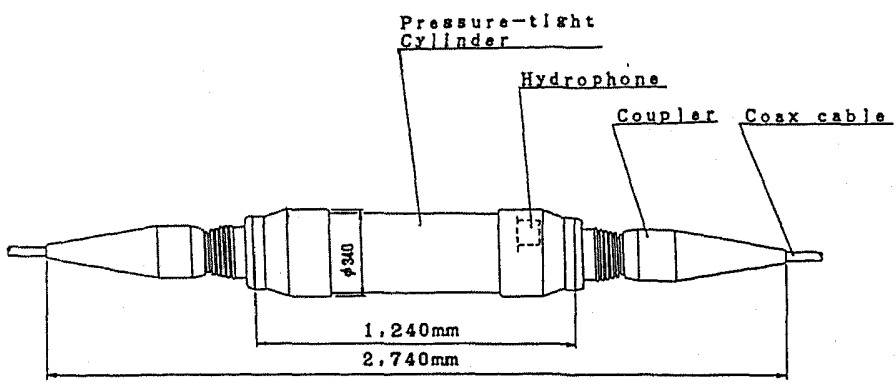

Fig. 2. Instrument to be deployed at $30^{\circ} \mathrm{N}$ on GeO-TOC cable.

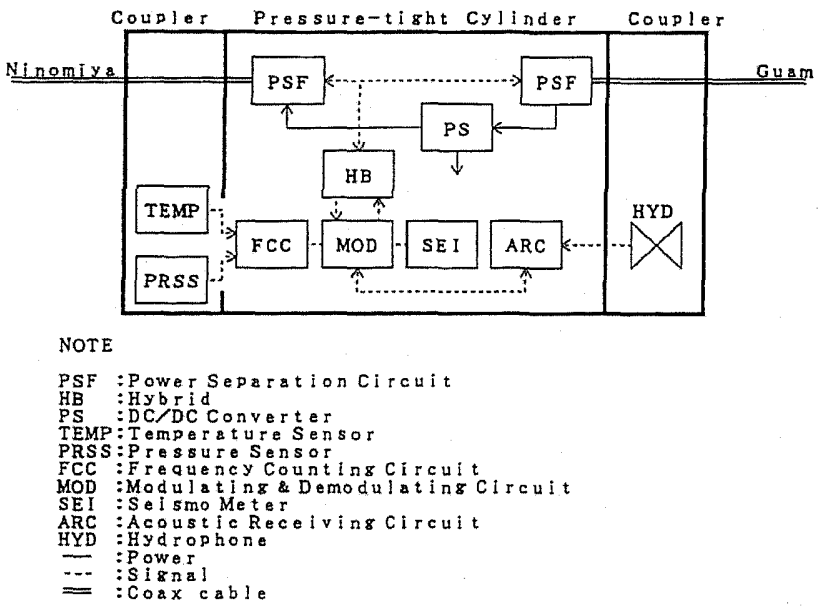

Fig. 3. Block diagram for GeO-TOC seismic instrument.

the necessary frequency resolution and necessary dynamic range, 24-bit Delta-Sigma A/D converters (CS5322/CS5323 manufactured by Crystal Semiconductor Co.) are used. These chips, however, are highly sensitive to temperature changes. By selecting electronic parts, the temperature drift due to $\mathrm{A} / \mathrm{D}$ converters is decreased to $1.4 \mathrm{mgal} /{ }^{\circ} \mathrm{C}$. In most commercial telephone cables, $4 \mathrm{kHz}$ frequency division is common. For the data acquisition controller, a 16-bit CMOS CPU (HD657348RCG, Hitachi Co.) was selected to minimize power consumption. Using $4 \mathrm{kHz}$ frequency division, commercial modem chips (MD96FX, Yamaha Co.) can be used. This chip can transmit data by many protocols (e.g., V.29, V.27). The transmission rate can be changed by control commands from the land. The total power dissipation is $30 \mathrm{~W}$ due to the loss in the power separation filter and the modem.

The instrument package can be deployed by methods similar to those use for repair work on submarine telephone cables (Suzuki, 1990). The cables in deep seas are hooked by dragging an anchor on the sea bottom perpendicular to the cable. After the cable is hooked by the anchor, the cable is cut and both cable ends are lifted to the ocean surface. On the ocean surface, each end is moored to a giant buoy. The instrument 
package with extra cable whose length is more than the water depth is connected to each end and is redeployed in the ocean. To do this installation work, it is better to use a normal cable-ship but the cost of a cable-ship is very high. Alternative method should be considered.

\section{Voltage Change Measurements Using the GeO-TOC Cable and Their Geophysical Implications}

A submarine telecommunication cable acts as a sensor on a planetary scale to measure geopotential variations (Lanzerotti, 1993). If the quasi-stationary or DC potential difference is measured, it might provide information on the dynamo process in the Earth's core (Runcorn, 1954). The voltage difference in the GeO-TOC connecting Guam and Ninomiya has been continuously measured since December 1991. The cable voltage is also affected by other kinds of phenomena, such as the total sea-water flux across the cable and electric field variations induced by geomagnetic field fluctuations, at shorter periods. Soon after TPC-1's retirement from communication service in 1991, the geopotential difference between Guam and Ninomiya was measured for approximately $8 \mathrm{~h}$ under an unpowered condition. Since that time, the whole cable system has been fed electric power of about $4,200 \mathrm{~V}$ and $370 \mathrm{~mA}$. Power needs to be fed to keep the cable system stable until its future reuse for ocean bottom observations. Therefore, the variations of the total supply voltage is being measured to monitor the shorter period components of natural geopotential variations. In the case of powered cable measurements, there are various kinds of problems (Larsen and Sanford, 1985), many of which are due to the effects of current fluctuations in the power feed system. In the present study, not only the supply voltage but also the supply current and the temperature of the power bay panel are recorded to correct the apparent voltage changes. The amplitude of the daily variations, one of the largest natural voltage changes, is approximately a few volts for this cable, which is smaller than the total voltage by nearly three orders of magnitude. A 6 and $1 / 2$ digit multimeter is used to measure these quantities with sufficient precision. The resolution of the voltage measurement is ca. $1 \mathrm{mV}$. Natural geopotential changes do not cause any current variations in the whole circuit because the resistance of the Earth is much lower than that of the cable circuit. After correcting the effects of temperature and supply current, the voltage variation record was analyzed, together with geomagnetic data at Guam and Kakioka, to obtain the magnetotelluric (MT) response as has already been reported (Fujii et al., 1993). In September 1993, another cable voltage measurement was initiated on the cable between Guam and Philippines (G-P), which lies almost in the E-W direction and nearly perpendicular to the GeO-TOC cable. This cable is used in an unpowered condition, and therefore the noise level of the voltage variation is quite low. A digital multimeter of the same type as for GeO-TOC measurements is used and therefore the voltage resolution is as small as $0.01 \mathrm{mV}$. The cable runs almost in the E-W direction, parallel to the geomagnetic equator. Figure 4 shows the simultaneous record of voltage variations for GeO-TOC and G-P. This shows the close resemblance between the two records. Comparing G-P data and data from HAW-1 between Hawaii and California, Fujii et al. (1995) reported interesting features of the short period potential variations

Vol. 43, No. 5, 1995 

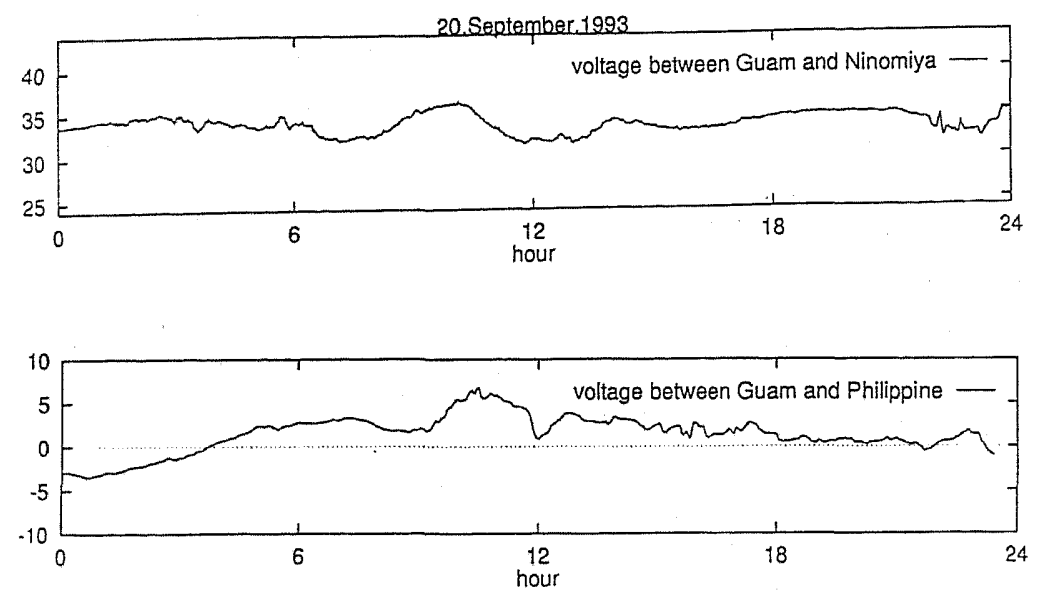

Fig. 4. Geopotential variation in GeO-TOC (top) and G-P (bottom) in September 29, 1993.

of ionospheric and magnetospheric origin.

When G-P measurements were started, a significant improvement was made to the measuring system. A data transmitter and a receiver were installed at Guam and Ninomiya, respectively, so that the data obtained in Guam could be monitored in Japan. The data transfer rate is $1,200 \mathrm{bps}$ with a sampling interval of $3 \mathrm{~s}$.

Using voltage data from these two orthogonally oriented cables, the regional electrical conductivity structure in the upper mantle beneath the Philippine Sea plate was investigated (Fujii et al., 1993). The geomagnetic field record at Muntinlupa in Luzon (Yumoto, personal communication), is helpful for the MT analysis of the G-P cable voltage.

\section{Future Plan to Reuse of Submarine Cable}

Scientific programs for reusing submarine coaxial cables have reached a new stage. There are other candidates for the reuse of cables in the Japan Sea and in the Philippine Sea. The former cable, called JASC (JApan Sea Cable) runs between Naoetsu, Japan and Nakhodka, Russia, and the latter, called TPC-2 runs between Okinawa, Japan and Guam. Both systems use coaxial cables (U.S. Department of Commerce, 1991).

TPC-2 was retired from service on April 1, 1994, and the Okinawa-Guam section is planned to be reused for the undersea environmental measurement, including seismic monitoring by the Science and Technology Agency (STA) of Japan. The power will be supplied from Okinawa and the Guam end will be grounded. The instrument to be attached to TPC-2 will be built in a different way from that of the GeO-TOC project. In the current plan, a bottom package will be deployed along the optical fiber cable attached to the coaxial cable using an ROV. This project is called VENUS (Versatile Eco-monitoring Network by Undersea-cable System).

JASC will be retired in 1995 under the cable companies' plan and after its retirement, 
it can be used for geophysical measurement by installing a bottom package at one end of the cable with small modifications. According to the plan of the cable companies, the Japan side the cable will be retrieved it at a water depth of $1,500 \mathrm{~m}$ from the landing station to the off-shore point. In the first phase of our plan, a sea-earth will be attached to this cut end and the electrical potential variation between this seaearth and Nakhodka end will be measured without feeding power.

A world-wide network of submarine cables for measuring geopotential distribution should be established to obtain information on the geodynamo process at the Earth's core. This is one of our future plans; wide-ranging international collaboration is needed. If such a network were to be established, significant contributions could be expected towards improving our understanding.

The idea of reusing old telephone cables was proposed by the late Mr. K. Kobayashi, who was deputy director of KDD Co., Ltd. and a similar idea was also proposed by Dr. S. Nagumo. Without their efforts, this project would not have been realized. The authors' thanks go to colleagues in the U.S.A. supporting the Guam GeO-TOC facilities. We also acknowledge that Prof. T. Yukutake, Kyusyu Univ. and Prof. N. Nasu, who was the chief scientific advisor to decide the route of TPC-1, encouraged the present project. This project has been supported by an earthquake prediction special program funded by the Ministry of Education, Science and Culture of Japan.

\section{REFERENCES}

Craig, Y., K. Horibe, A. Farley, J. A. Welhan, K.-R. Kim, and R. N. Hey, Hydrothermal vents in the Mariana trough; results of the first ALVIN dives, Eos Trans., $A G U$, 68, $1531,1987$.

Dziewonski, A. M., Mapping of the lower-mantle-determination of lateral heterogeneity in P velocity up to degree an order 6, J. Geophys. Res., 89, 5929-5952, 1984.

Fujii, I., H. Utada, Y. Hamano, and T. Yukutake, Apparent resistivity from the voltage variations measured on the submarine cable between Guam and Ninomiya, 14th General Assembly IAGA, 1993.

Fujii, I., L. J. Lanzerotti, H. Utada, H. Kinoshita, J. Kasahara, L. V. Medford, and C. G. Maclennan, Geoelectric power spectra over oceanic distances, Geophys. Res. Lett., 22, 421-424, 1995.

IRIS Steering Committee for Scientific Use of Submarine Cables, Scientific use of submarine telecommunications cables, Eos Trans., $A G U, 73(9), 97$, 100-101, 1992.

Kasahara, J., Engineering model of TPC-1 project, in Workshop on Scientific Uses of Undersea Cables, ed. A. Chave and T. Pyle, pp. 266-273, Joint Oceanographic Inst., Inc., Washington, D.C., 1990.

Kasahara, J. and K. Tanaka, Visual Seismology, Univ. Tokyo Press, Tokyo, pp. 26-29, 1984 (in Japanese).

Kasahara, J., L. Kong, S. Koresawa, C. Igarashi, T. Yamaguchi, M. Suzuki, and R. Feng-Lu, Seismic observation at the Mariana trough at $18^{\circ} \mathrm{N}$ and its relation to the detailed bathymetry, Preliminary Report of the Hakuho-Maru Cruise KH92-1, Ocean Research Institute, Univ. Tokyo, 77-98, 1993.

Katao, H., J. Kasahara, and S. Koresawa, Seismic observations using inertial navigation servo

Vol. 43, No. 5,1995 
accelerometers for application to the broad-band ocean bottom seismometer, Bull. Earthq. Res. Inst., Univ. Tokyo, 65, 633-648, 1990 (in Japanese).

Lanzerotti, L. J., A. D. Chave, C. H. Sayres, L. V. Medford, and C. G. Maclennan, Large-scale electric field measurements on the Earth's surface: a review, J. Geophys. Res., 98, 23525-23534, 1993.

Larsen, J. C. and T. B. Sanford, Florida current volume transport from voltage measurements, Science, 227, 302-304, 1985.

Runcorn, S. K., The Earth's core, Eos Trans. AGU, 35, 49-63, 1954.

Suzuki, K., Design consideration on transmission capacity, power feeding, and laying method for the reuse of TPC-1 cable for scientific purpose, in Workshop on Scientific Uses of Undersea Cables, ed. A. Chave and T. Pyle, pp. 277-289, Joint Oceanographic Inst., Inc., Washington, D.C., 1990.

U. S. Department of Commerce, 1990 World's submarine telephone cable systems, NTIA-CR91-42, 1991. 